\title{
BIMAXILLARY PROTRUSION - A CASE REPORT
}

Hemant Kumar Halwai, ${ }^{1}$ Sumit Kumar Yadav, ${ }^{1}$ Kishor Dutta, ${ }^{1}$ Sandeep Kumar Gupta, ${ }^{1}$ Raju Shrestha, ${ }^{1}$ Anish Kumar Shah ${ }^{1}$

\begin{abstract}
Correction of a severe bimaxillary protrusion with maximum anchorage can be challenging. This case report describes the treatment of a girl with a bimaxillary protrusion. Orthodontic treatment included extraction of her 4 first premolars. The total treatment time was 18 months. Her dental proclination and facial appearance was significantly improved.
\end{abstract}

KEYWORDS Bimaxillary protrusion, extraction, premolars, profile

1. Department of Orthodontics and Dentofacial Orthopedics, UCMS College of Dental Surgery, Bhairahawa, Nepal

DOI: https://doi.org/10.3126/jucms.v7i1.24697

\author{
For Correspondence \\ Dr. Hemant Kumar Halwai \\ Department of Orthodontics and Dentofacial Orthopedics \\ UCMS College of Dental Surgery, Bhairahawa, Nepal \\ E-mail: drhemanthalway@gmail.com
}




\section{INTRODUCTION}

Bimaxillary protrusion is a condition characterized by protrusive and proclined upper and lower incisors and an increased procumbency of the lips. ${ }^{1}$ It is seen commonly in African-American ${ }^{2}$ and Asian ${ }^{3}$ populations, but it can be seen in almost every ethnic group. Because of the negative perception of protrusive dentition and lips in most cultures, many patients with bimaxillary protrusion seek orthodontic care to decrease this procumbency.

The etiology of bimaxillary protrusion is multifactorial and consists of a genetic component as well as environmental factors, such as mouth breathing, tongue thrusting, lip biting habits, and tongue volume. ${ }^{3}$

The goals of orthodontic treatment of bimaxillary protrusion include the retraction and retroclination of maxillary and mandibular incisors with a resultant decrease in soft tissue procumbency and convexity. The successful orthodontic correction of bimaxillary protrusion has been reported. ${ }^{4.5}$ Tan studied orthodontic correction of bimaxillary protrusion in 50 Chinese adult patients and found favorable soft tissue and dental changes after the extraction of four premolars. ${ }^{6}$ In a case report on the use of four premolar extraction and lingual appliances for the corrections of bimaxillary protrusion, $\mathrm{Kurz}^{7}$ found that the upper and lower incisors became more retroclined and retrusive, resulting in a greatly improved facial pro?le.

\section{CASE PRESENTATION}

A 22-year-old girl reported to department of Orthodontics, UCMS College of Dental Surgery with a chief complaint of forwardly placed upper and lower front teeth. There was no history of dental trauma or oral habits. The patient had good oral hygiene. Her medical history showed no contraindication to orthodontic treatment.

\section{DIAGNOSIS}

Patient had a convex pro?le with orthognathic maxilla and orthognathic mandible. She had procumbent upper and lower lips (Figure 1). Her dentition was characterized by a Class I malocclusion with bimaxillary dental proclination (Figure 1 and 2). Panoramic radiograph showed presence of 30 teeth with missing maxillary $3^{\text {rd }}$ molar and with no evidence of any bony loss (Figure 3). The lateral cephalometric radiograph showed ANB angle of $2^{\circ}$, indicative of Class I skeletal jaw bases (Figure 3). As evidenced by Frankfort-mandibular plane angle of $28^{\circ}$, skeletal pattern was average growth pattern.

The patient had proclined maxillary and mandibular incisors with UI-NA $10 \mathrm{~mm} / 42^{\circ}$ and L1-NB $9 \mathrm{~mm} / 32^{\circ}$ (Table 1 ).
Table 1. Cephalometric Appraisal

\begin{tabular}{cccc}
\hline Parameter & Normal & Pretreatment & Post treatment \\
\hline SNA & $82^{\circ}$ & $82^{\circ}$ & $81.5^{\circ}$ \\
\hline SNB & $80^{\circ}$ & $80^{\circ}$ & $79.5^{\circ}$ \\
\hline ANB & $2^{\circ}$ & $2^{\circ}$ & $2^{\circ}$ \\
\hline FMA & $25^{\circ}$ & $28^{\circ}$ & $28^{\circ}$ \\
\hline U 1 to NAmm / deg & $4 \mathrm{~mm} / 22^{\circ}$ & $10 \mathrm{~mm} / 42^{\circ}$ & $5 \mathrm{~mm} / 24^{\circ}$ \\
\hline L 1 to NBmm / deg & $4 \mathrm{~mm} / 25^{\circ}$ & $9 \mathrm{~mm} / 32^{\circ}$ & $4 \mathrm{~mm} / 25^{\circ}$ \\
\hline IMPA & $90^{\circ}$ & $100^{\circ}$ & $92^{\circ}$ \\
\hline Nasolabial angle & $102^{\circ}$ & $92^{\circ}$ & $100^{\circ}$ \\
\hline Upper lip to S line & $0 \mathrm{~mm}$ & $3 \mathrm{~mm}$ & $0.5 \mathrm{~mm}$ \\
\hline Lower lip to S line & $0 \mathrm{~mm}$ & $5 \mathrm{~mm}$ & $0 \mathrm{~mm}$ \\
\hline
\end{tabular}

\section{Treatment Objectives}

The primary objective was to correct bimaxillary dental proclination and lip procumbency. Treatment objectives for the occlusion were to maintain the molar neutrocclusion, to achieve ideal overjet, overbite and achieve canine guidance.

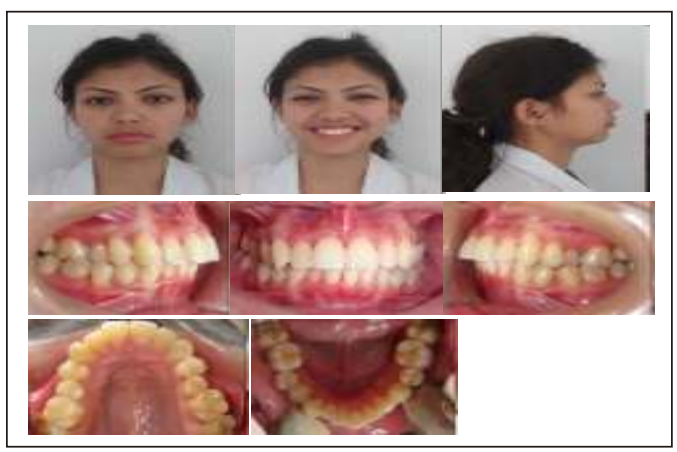

Figure1. Pre-treatment extra-oraland intra-oral photographs

The main issue in determining the appropriate treatment plan was the severity of dentoalveloar protrusion. It was recommended that the 4 first premolars be extracted to reduce the patient's lip procumbency. Another treatment alternative was a non-extraction plan with interproximal tooth reduction of the premolars. This plan would not address the patient's chief complain. With reproximation, the incisal angulations would not be affected, and the patient's bimaxillary protrusion would remain the same.

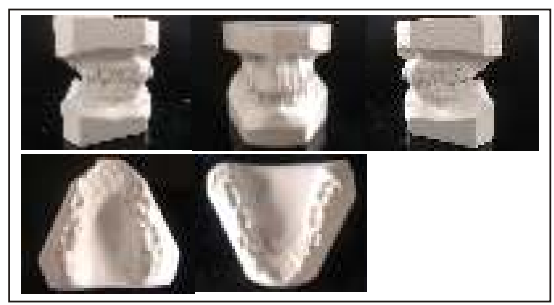

Figure 2. Pretreatment study models 

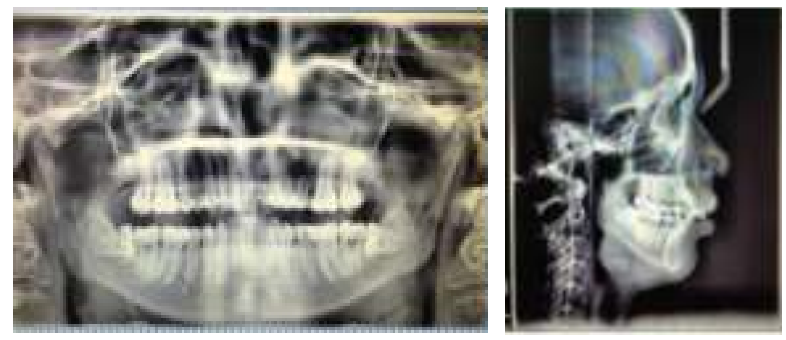

Figure 3. Pretreatment radiographs

\section{Treatment Progress}

MBT appliance $0.022 \times 0.028$ " slots was used. A transpalatal arch in maxilla and lingual arch in mandible was placed on banded 1st molars to enhance the anchorage. Alignment and leveling was accomplished with following sequence of arch wires: (a) 0.016 "nickel-titanium arch wires (b) 0.018 "stainless steel arch wires and (c) $0.017 \times 0.025^{\prime \prime}$ stainless steel wires. The arch wires were cinched distal to molar to avoid maxillary and mandibular incisor proclination. After aligning and levelling, the maxillary and mandibular dentition was consolidated on $0.017 \times 0.025^{\prime \prime}$ stainless steel wire. The en masse retraction was accomplished by sliding mechanics using $9 \mathrm{~mm}$ NiTi coil spring on $0.019 \times 0.025^{\prime \prime}$ stainless steel wire. The NiTi coil spring delivered 150 grams of continuous force without any permanent deformation.Finishing and detailing was carried out by $0.021 \times 0.025$ "stainless steel wire. Upper and lower retainers were placed and case debonded. The treatment was finished in eighteen months. The patient was given a maxillary and mandibular anterior bondable lingual retainer. The patient was recalled for follow up every six months, but patient did not come for follow up.

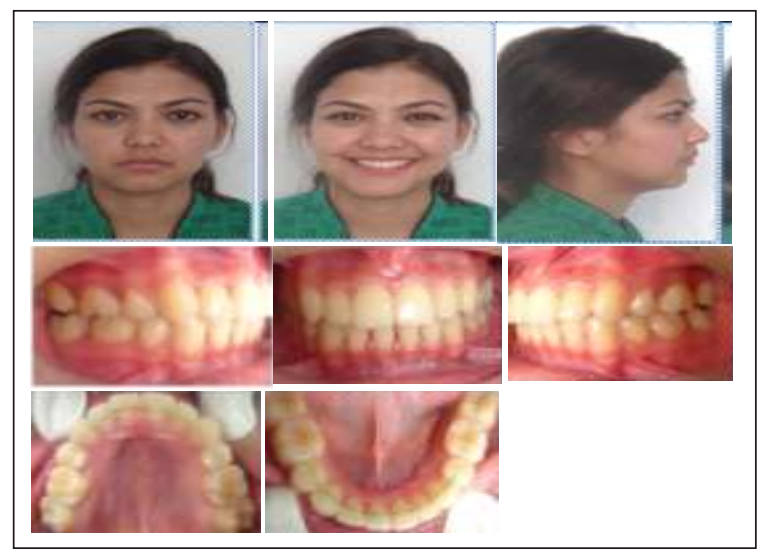

Figure 4. Post-treatment extra-oral and intra-oral photographs

\section{Treatment Result}

The change in the patient's facial esthetics was the most impressive part of her treatment. With extraction of the $1^{\text {st }}$ premolars, $5 \mathrm{~mm}$ retraction of upper and lower anterior teeth was achieved. Her lip incompetency was reduced; nasolabial angle and mentolabial sulcus improved (Figure 4).The molar relation and vertical dimension were maintained during orthodontic treatment (Figures 4 and 5). Post treatment intraoral photographs and lateral cephalogram showed that the maxillary and mandibular incisors were inclined appropriately (Figures 4 and 6). The panoramic radiograph showed adequate root parallelism in both upper and lower arches (Figure 6).

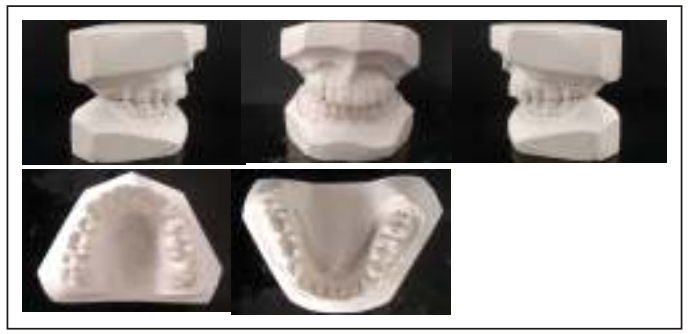

Figure 5. Post-treatment study models
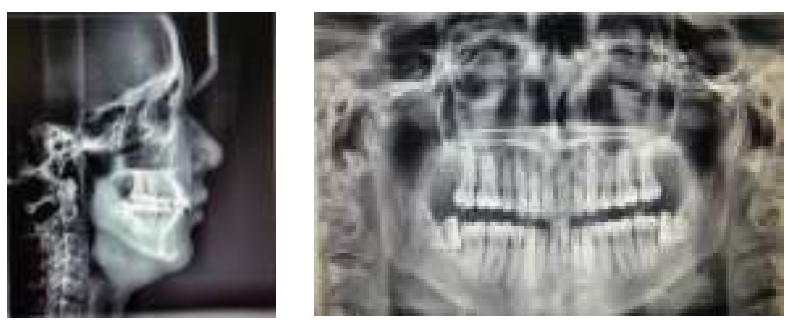

Figure 6. Post-treatment radiographs

\section{DISCUSSION}

Bimaxillary protrusion is common among various ethnic groups, the most affected population being Asians and American of African descent. ${ }^{3}$ It is characterized by severe proclination of anterior teeth of both the arches, with a resultant increase in lip procumbency. The treatment protocol includes extraction of ? rst premolars to correct dental proclination and to reduce lip incompetency. Drobocky and Smith revealed that almost all patients treated with extraction of ? rst premolars have an average reduction of $3.4 \mathrm{~mm}$ and 3.6 $\mathrm{mm}$ in upper and lower lip procumbency in relation to Rickett's E-line. ${ }^{8}$ When premolars are extracted to correct the malocclusion, the treatment plan must account for closure of extraction space.

The main challenges confronted by the orthodontist are anchorage maintenance, since mesialization of the posterior 
segment may compromise retraction of anterior teeth. Andreasen Gf ${ }^{9}$ have reported a range of mesial molar movement of 0 to $2.4 \mathrm{~mm}$ when retraction is combined with the use of adjunctive appliances to control anchorage. Maximum anchorage has been considered vital in such cases. In our case, we used transpalatal arch given by Goshgerian; it is economical, easy to fabricate, and the most reliable method to augment anchorage ${ }^{10}$.

MBT appliance was used in this case because this prescription can achieve excellent force levels and resulting in tooth movement with excellent control of the biomechanics during the space closure of the extraction sites. ${ }^{11}$

\section{CONCLUSION}

In this patient with procumbent upper and lower lips, excessive lip strain, proclined maxillary and mandibular incisors, an acceptable treatment result was obtained with 4first-premolars extraction plan.

The patient's profile was improved, with reduction in lip procumbency and decrease in lip protrusion. The interincisal angulation improved significantly because both the maxillary and the mandibular incisors were uprighted after space closure.

\section{REFERENCES}

1. Bills, Daniel A., Chester S. Handelman, and Ellen A. BeGole. "Bimaxillary dentoalveolar protrusion: traits and orthodontic correction." The Angle Orthodontist.2005; 75:333-339.

2. Farrow AL, Zarrinnia K, Azizi K. Bimaxillary protrusion in black Americans-an esthetic evaluation and the treatment considerations. Am J Orthod Dentofacial Orthop. 1993;104 (3): 240-50.

3. Lamberton CM. Bimaxillary Protrusion as a pathologic problem in the Thai. Am J Orthod. 1980;77(3):320-29.

4. Diels RM, Kalra V, DeLoach Jr N, Powers M, Nelson SS. Changes in soft tissue profile of African-Americans following extraction treatment. The Angle Orthod 1995 Aug; 65:285-92.

5. Kocadereli I. Changes in soft tissue profile after orthodontic treatment with and without extractions. Amj Dentofacial Orthopedics. 2002 Jul 1;122 (1):67-72.

6. Tan TJ. profile changes following orthodontic correction of bimaxillary protrusion with a preadjusted edgewise appliance. Int J Adult orthodon orthognath surg, 1996;11:239-251.
7 Kurz C. American Journal of orthodontics and dentofacial orthopedics The use of lingual appliances for correction of bimaxillary protrusion ( four premolars extraction ). Am J Orthod Dentofacial Orthop. 1997;112(4):357-363.

8 Drobocky OB, Smith RJ. changes in facial profile during orthodonitcs treatment with extraction of four first premolars. Am j orthod dentofacial orthop. 1989;95(3):220-30.

9 Andreasen GF, Zwanziger D. A clinical evaluation of diferential force concept as applied to the edgewise bracket. Am J Orthod. 1980;78:25-40.

10 Stivaros N, Lowe C, Dandy N, Doherty B, Mandall NA. A randomized clinical trial to compare the Goshgarian and Nance palatal arch. The European Journal of Orthodontics. 2010; 32(2):171-176.

11 Mclaughlin, Bennett, Trevisi. systemized orthodontic treatment mechanics. Mosby publisher limited, 2001. 